\title{
Clinical features and long-term outcomes of moyamoya disease: a single-center experience with 528 cases in China
}

\author{
*Xing-ju Liu, MD,, Dong Zhang, MD,1 Shuo Wang, MD,' Yuan-li Zhao, MD,1 \\ Mario Teo, MD, FRCS(SN), ${ }^{1,2}$ Rong Wang, MD, ${ }^{1}$ Yong Cao, MD, ${ }^{1}$ Xun Ye, MD, ${ }^{1}$ Shuai Kang, MD, ${ }^{1}$ \\ and Ji-Zong Zhao, MD'1
}

\begin{abstract}
1'Department of Neurosurgery, Beijing Tiantan Hospital, Capital Medical University, Beijing, China; and 'Department of Neurosurgery, Institute of Neurological Science, Glasgow, United Kingdom
\end{abstract}

\begin{abstract}
OBJECT The aim of this study was to describe the baseline clinical features and long-term outcomes of patients with moyamoya disease (MMD) based on a 25 -year period at a single center in China.

METHODS Data obtained in 528 consecutive patients with MMD treated at the authors' hospital from 1984 to 2010 were reviewed retrospectively. Events of transient ischemic attack, new infarction, and hemorrhage were included. The Kaplan-Meier risk of stroke was calculated.
\end{abstract}

RESULTS The mean ( \pm SD) patient age was $26 \pm 13$ years (range 2-67 years), and the female/male ratio was 0.9:1. There were 332 cases of ischemia and 196 hemorrhages. Adults had a higher rate of bleeding than children $(50.7 \%$ vs $14.0 \%$, respectively; $p<0.001$ ). One hundred twenty-two patients were treated conservatively, and 406 patients underwent revascularization procedures. Of 528 patients, 331 (62.7\%) had at least 1 year of follow-up (median 39.5 months) and data from these patients were analyzed. Rebleeding and mortality rates in patients with hemorrhagic MMD $(n=$ 104) were higher than in those with ischemic MMD $(n=227)(26.9 \%$ vs $2.2 \%[p<0.001]$ and $4.8 \%$ vs $0.4 \%[p<0.05]$, respectively). Twenty-five of $60(41.7 \%)$ conservatively treated patients and 8 of $271(2.9 \%)$ surgically treated patients experienced rebleeding events, a difference that was significant in the Kaplan-Meier curve of rebleeding $(p<0.01)$. An improvement in perfusion was found in 164 of 224 (73.2\%) surgically treated patients 1 month after discharge. However, there was no significant difference in the rate of ischemic events in the surgical and conservative groups (18.8\% and $28.3 \%$, respectively; $p=0.09$ ). Among the 104 hemorrhagic cases, rebleeding attacks were observed in 25 patients in the nonsurgical group $(n=60)$ and 3 patients in the surgical group $(n=44)(41.7 \%$ and $6.8 \%$, respectively; OR 9.7 [95\% Cl 2.7-35.0]; $p<0.01)$.

CONCLUSIONS There was no difference in the sex distribution of Chinese patients with MMD. Patients with hemorrhagic MMD had a much higher rate of rebleeding and poorer prognosis than those with the ischemic type. Surgical revascularization procedures can improve cerebral perfusion and have a positive impact in preventing rebleeding in patients with hemorrhagic MMD.

http://thejns.org/doi/abs/10.3171/2014.10.JNS132369

KEY WORDS moyamoya disease; China; hemorrhage; revascularization; vascular disorders

$\mathrm{M}$ OYAMOYA disease (MMD), an uncommon chronic cerebrovascular disease, was first reported by Takeuchi and Shimizu in 1957.24 The pathology of the disease is characterized by progressive narrowing or occlusion of the bilateral distal internal carotid arteries with abnormal vascular collateral networks at the base of the brain. In 1969, Suzuki and Takaku named it "moyamoya disease" according to the angiographic appearance of a hazy "puff of smoke." 22 There are 2 main phenotypes of MMD in Asian populations: the ischemic type, which is common in children, and the hemorrhagic type, which is common in adults. ${ }^{7,17}$ At present, the pathophysiology of

\footnotetext{
ABBREVIATIONS EDAS = encephaloduroarteriosynangiosis; MMD = moyamoya disease; $m R S=$ modified Rankin Scale; STA-MCA = superficial temporal artery to middle cerebral artery; TIA = transient ischemic attack.

SUBMITTED October 25, 2013. ACCEPTED October 14, 2014.

INCLUDE WHEN CITING Published online November 28, 2014; DOI: 10.3171/2014.10.JNS132369

DISCLOSURE The authors report no conflict of interest concerning the materials or methods used in this study or the findings specified in this paper. The following grants funded this study: National Science and Technology supporting plan grant no. 2006BAl01A13 (the "11th Five-Year Plan") and Beijing Municipal S\&T Commission grant no. D101107049310001.

* Drs. Liu and Zhang contributed equally to this work.
} 
MMD is poorly understood, and guidelines for the standard treatment of MMD have still not been constituted.

Moyamoya disease appears to be relatively rare in Western countries. In Asia, however, it is a common cause of stroke in patients younger than 50 years. Recently, more and more cases of MMD have been detected in China. According to recent data, the incidence of MMD in the Chinese population does not seem to be any lower than that in the Japanese population. ${ }^{20}$ However, there is a lack of reports regarding the clinical characteristics, natural history, and long-term outcome of patients with this condition in the Chinese population. Here, we retrospectively review the demographic data, clinical features, treatments, and long-term outcomes of patients treated at our institution over the past 25 years.

\section{Methods}

\section{Patient Selection}

Patients with a clinical diagnosis of MMD were identified from the database of our Cerebrovascular Neurosurgery Center from January 1984 to December 2010. Cerebral digital subtraction angiography or MR angiography was carried out for all the patients. The diagnostic criteria for MMD were based on the guidelines reported in 1995 by Fukui: $^{7}$ 1) stenosis or occlusion of the terminal internal carotid and the proximal middle and anterior cerebral arteries and 2) unilateral or bilateral involvement. Patients with any other disease that might explain the arterial steno-occlusive disease were excluded.

\section{Retrospective Chart Review}

This study was approved by the Beijing Tiantan Hospital Research Ethics Committee. We reviewed the clinical records, including hospital charts, clinic notes, and radiographic data. At initial presentation, we noted baseline stroke risk factors, including hypertension, cerebral aneurysm, familial MMD, hyperlipidemia, diabetes mellitus, hyperthyroidism, hypertensive heart disease, significant alcohol and/or tobacco use, oral contraceptive use, and parental stroke history. Until now, there have been no clear guidelines for the surgical treatment of MMD; therefore, in our series, the selection of surgical treatment for MMD was based mainly on 3 criteria (nonemergency status, symptoms of ischemia or hemorrhage, and the patient's condition warranting surgery), whereas decisions to perform revascularization procedures were based on the age of the patients and the condition of the intra- and extracranial arteries. According to the treatments, patients were categorized as having had conservative treatment or revascularization surgery. Revascularization surgery included bypass of the superficial temporal artery to the middle cerebral artery (STA-MCA), encephaloduroarteriosynangiosis (EDAS), multiple bur holes, and carotid artery adventitial denudation (also known as bilateral cervical perivascular sympathectomy, which was first reported by Suzuki et al. for the treatment of children $\mathrm{MMD}^{23}$ ).

\section{Clinical Follow-Up}

For each patient, an outcome assessment was performed using the modified Rankin Scale (mRS) at discharge and follow-up. CT perfusion or SPECT was used to assess the changes in cerebral blood flow of patients in the surgical revascularization group at the 1st and 3rd postoperative months. The average radioactivity was calculated, and cerebellar blood flow was used as the normal reference. We compared the ratio of the same regional cerebral blood flow to the cerebellar blood flow before and after the operation for each patient. An increase or decrease in this ratio was used to identify any improvement or deterioration, respectively, of cerebral blood flow. The following events were included: transient ischemic attack (TIA), new infarction, and hemorrhage. Because most of the early patients did not undergo a CT perfusion examination, and some patients had less than 1 year of follow-up, we analyzed only the long-term follow-up data for a subgroup of 331 patients who were admitted between December 2000 and December 2010 (Fig. 1).

\section{Statistical Analysis}

The data were analyzed statistically using SPSS for Windows, version 16.0 (SPSS, Inc.). Continuous and categorical data were compared with the t-test and chisquare test, respectively. Stroke-free survival analysis was performed using Kaplan-Meier curves, with comparisons made using log-rank statistics. A p value of $<0.05$ was considered statistically significant.

\section{Results}

\section{General Patient Characteristics}

We identified 528 cases of MMD at Beijing Tiantan Hospital between January 1984 and December 2010. The median $( \pm$ SD) patient age was $26 \pm 13$ years (range 2-67 years). The number of new patients diagnosed with MMD in our hospital has increased significantly year by year (Fig. 2). There were 282 men and 246 women, for a female/male ratio of $0.9: 1$. Two definite peaks in age distribution were found for the combined population of male and female patients (Fig. 3 left).

The initial symptom was ischemia or hemorrhage in 332 and 196 patients, respectively. The majority of ischemic patients presented with TIA; infarction was the second most common presentation for these patients. In patients with hemorrhagic MMD, intraventricular hemorrhage was the most common type, and intracerebral hemorrhage was the second most common. Adults had a higher rate of bleeding than children $(50.7 \%$ vs $14.0 \%$, respectively; OR 3.60 [95\% CI 2.51-5.16]; $p<0.001$ ), whereas ischemia occurred more often in pediatric patients than in adults (Fig. 3 right). Most patients presented with Suzuki and Takaku angiographic Stage 3 or 4 MMD (58.2\%), and 41 (7.7\%) patients had unilateral disease. There was no significant difference in angiographic stage between hemorrhagic and ischemic patients $(\mathrm{p}>0.05)$. Patient characteristics are detailed in Tables 1 and 2.

\section{Baseline Stroke Risk Factors}

The baseline stroke risk factors for all patients were established. There were 40 patients with hypertension, 28 with a history of cerebral infarction, 16 with cerebral aneurysm, 10 with diabetes, 47 with a history of smoking 


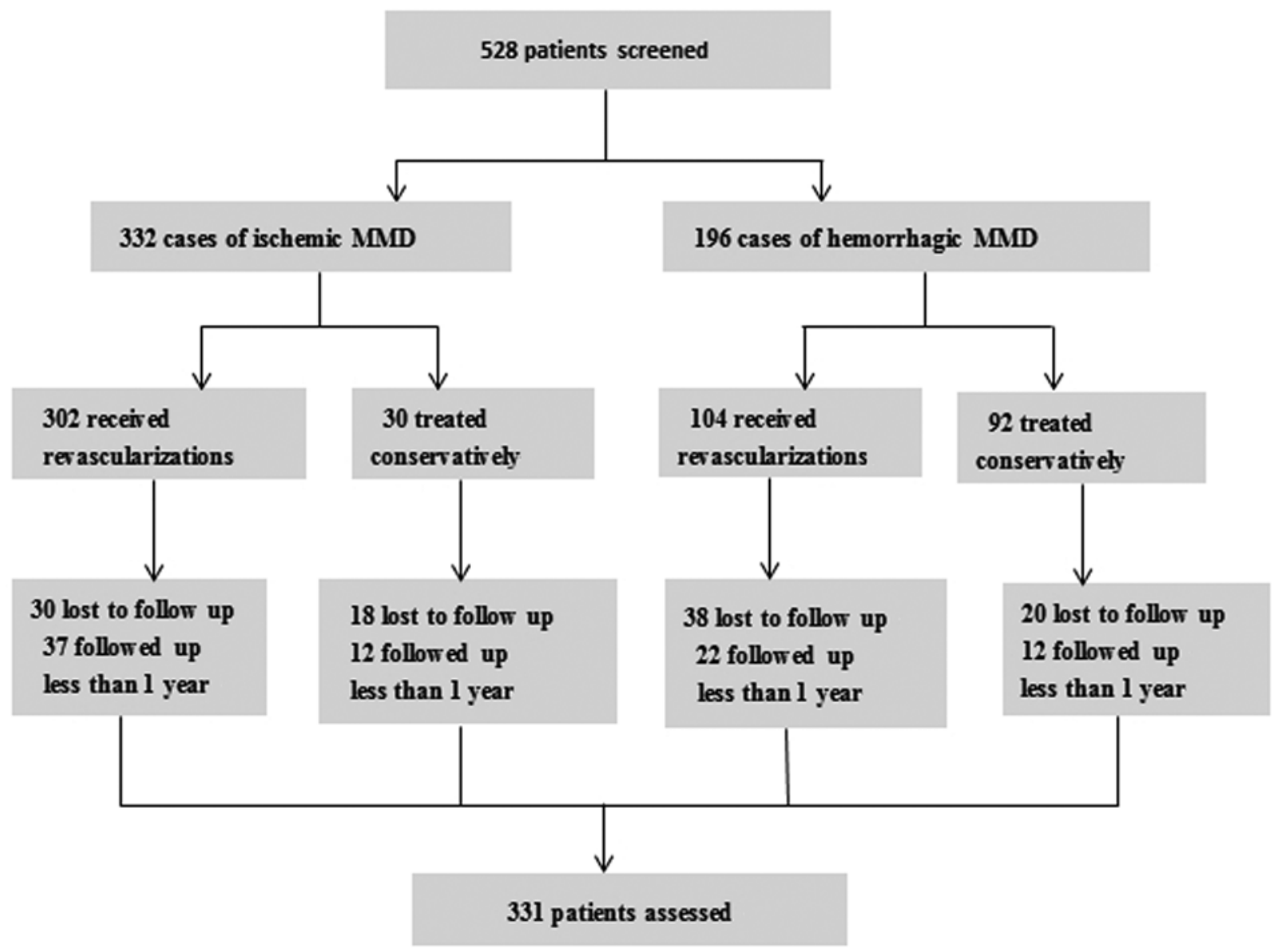

FIG. 1. Flow chart of the patient cohort with presentations, management strategies, and follow-up.

or alcohol use, 5 with hyperthyroidism, 20 with sinus arrhythmia, 14 with sinus tachycardia or bradycardia, and 9 with familial MMD. The proportion of patients with a cerebral aneurysm in the hemorrhagic group was higher than that in the ischemic group $(\mathrm{p}<0.01)$. No significant differences in the other risk factors were observed between hemorrhagic and ischemic patients (Table 2).

\section{Treatment and Outcome}

One hundred twenty-two (23.1\%) of the 528 patients were treated conservatively, and $92(75.4 \%)$ of them had hemorrhagic MMD. However, of these patients, 8 received craniotomies to evacuate an intracranial hematoma, 3 received external ventricular drains, and 2 received ventriculoperitoneal shunts. Of 13 patients with an aneurysm, 5 underwent aneurysm clipping, 2 had aneurysms that disappeared after revascularization, and 6 were treated conservatively. Of the 122 patients treated conservatively, 2 patients died of cerebral hemorrhage during hospitalization, 10 patients had severe disability (mRS score $>3$ ), and 31 patients had slight to moderate disability $(2 \leq \mathrm{mRS}$ score $\leq 3$ ) at discharge.

Of the 406 patients who underwent surgical revascularization, 186 (45.8\%) were treated by direct-bypass STAMCA anastomosis, including 15 patients who received bilateral procedures. Twenty-six patients underwent an STA-MCA bypass combined with indirect revasculariza- tion. Intraoperative indocyanine green angiography was performed in 176 patients. The remaining 194 patients underwent indirect revascularization, including 72 in whom EDAS was performed, 76 who received multiple cranial bur holes, and 46 who received carotid artery adventitial denudation.

In the 186 patients treated with an STA-MCA bypass, postoperative complications included 2 cerebral infarctions, 4 intracranial hemorrhages, 2 seizures, and 5 wound infections. In the 194 patients treated by indirect revascularization, there were 5 subdural hydromas and 4 seizures. No patient died as a result of postoperative complications. For the 186 patients treated by STA-MCA bypass, cerebral blood flow studies were performed in 156 patients at discharge; 117 patients (75\%) showed an increase in cerebral perfusion, 21 patients showed no change, and 8 patients showed a decrease. According to $\mathrm{mRS}$ scores, 4 patients $(2.2 \%)$ had severe disability ( $\mathrm{mRS}$ score $>3$ ), and 18 patients $(9.7 \%)$ had slight to moderate disability $(2 \leq \mathrm{mRS}$ score $\leq 3)$ at discharge. There were no significant differences in $\mathrm{mRS}$ scores between the indirect revascularization and STA-MCA bypass groups $(\mathrm{p}=0.282)$.

\section{Follow-Up}

In total, 331 patients $(62.7 \%)$ had at least 1 year of follow-up. The median follow-up time was 39 months (range 12-300 months). Of these patients, the initial symptom of 


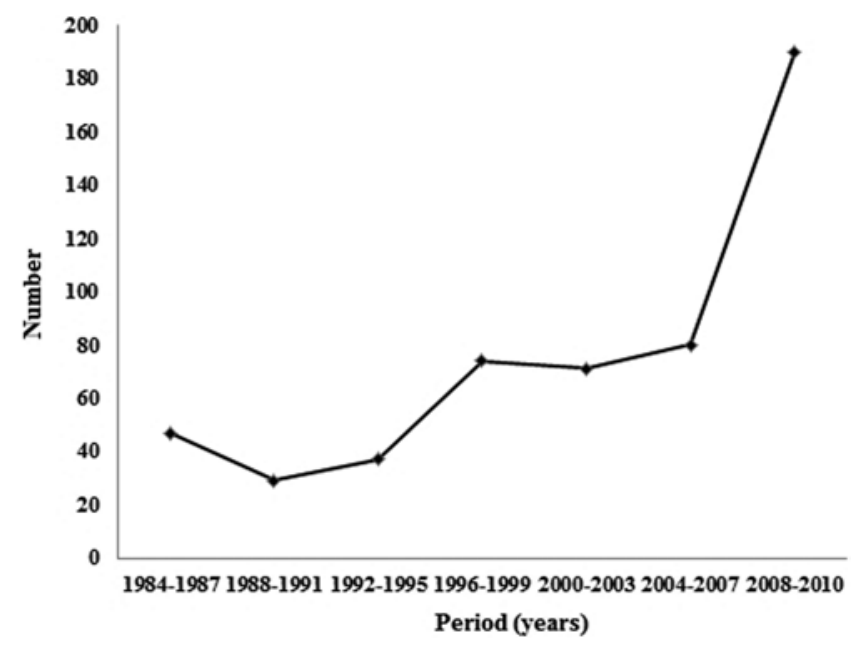

FIG. 2. Distribution of the number of new admissions for patients with MMD. The number of MMD cases has increased dramatically in recent years.

presentation was ischemia in 227 and hemorrhage in 104. Sixty patients who received conservative management had hemorrhagic MMD. The remaining 271 patients underwent neurosurgical revascularization procedures, including indirect bypass in 94 patients and STA-MCA bypass in 177 patients.

There were 33 intracranial hemorrhages (9.9\%), 68 TIAs, and 43 new infarctions during the follow-up period. Twenty-five of $60(41.7 \%)$ conservatively treated patients and 8 of $271(2.9 \%)$ surgically treated patients experienced a rebleeding event (OR 23.4; 95\% CI 9.8-56.1; $\mathrm{p}<0.01$ ). This finding was statistically significant in the KaplanMeier curve of rebleeding between the 2 groups (Fig. 4 left). Patients with hemorrhagic MMD had a significantly higher rate of rebleeding than patients with ischemic MMD (26.9\% vs $2.2 \%$, respectively; $\mathrm{p}<0.001$ ) (Fig. 4 right). Two hundred sixty-seven patients underwent brain perfusion scanning. Of these, an improvement in cerebral perfusion was found in $164(73.2 \%)$ of 224 surgically treated patients and $7(16.3 \%)$ of 43 conservatively treated patients 1 month after discharge (OR 14.1; 95\% CI 5.9-33.2; $\mathrm{p}<0.01$ ). There were significant differences in the mRS scores between the surgically and conservatively treated patients and between the ischemic and hemorrhagic patients at the time of the latest follow-up. The risk of ischemic events in the surgical group was $18.8 \%$, lower than that for the conservative treatment group (28.3\%); however, this difference was not statistically significant (Table 3).

Of the 104 hemorrhagic patients, 44 patients underwent surgical revascularization, including STA-MCA anastomoses in 25, STA-MCA anastomoses combined with EDAS in 4, EDAS in 6, multiple bur holes in 7, and carotid artery adventitial denudations in 2 . Rebleeding was observed in $25(41.7 \%)$ of 60 patients treated conservatively and in $3(6.8 \%)$ of 44 patients who underwent surgical revascularization (1 was treated with STA-MCA bypass, 1 with EDAS, and 1 with multiple bur holes). The rebleeding ratio in patients treated conservatively was significantly higher than that in patients who underwent surgical revascularization (OR 9.7; 95\% CI 2.7-35.0; $\mathrm{p}<0.01$ ).

\section{Discussion}

This large cohort study extends our knowledge of the clinical features, surgical treatment, and long-term outcome of patients with MMD in mainland China. There were mainly two classic presenting symptoms: intracranial hemorrhage, which commonly occurred in adults, and ischemic stroke, which mostly occurred in children. Ischemic symptoms were more common than hemorrhagic symptoms. There were also 2 peaks for the age of onset in Chinese patients with MMD. These epidemiological features of MMD in Chinese patients are similar to those found in reports from Japan and South Korea. ${ }^{11,17,25}$ In addition, we also found a definite increase in the number of
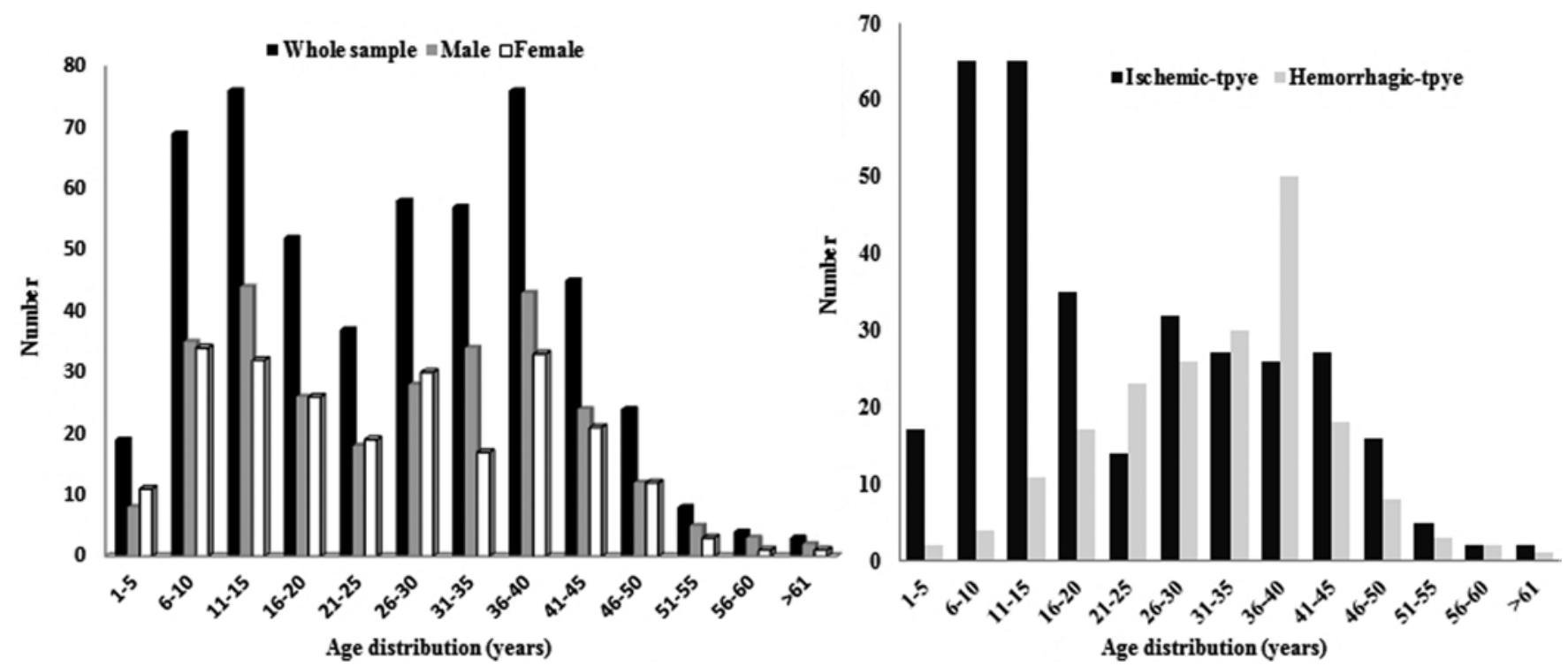

FIG. 3. Left: Age distribution of the patients. Right: Age distribution of patients with hemorrhagic MMD compared with ischemic MMD. 
TABLE 1. Patient characteristics

\begin{tabular}{|c|c|}
\hline Characteristic & No. of Patients (\%) \\
\hline No. of patients & 528 \\
\hline Female/male ratio & $246 / 282$ \\
\hline \multicolumn{2}{|l|}{ Age } \\
\hline$\leq 18 \mathrm{yrs}$ & $199(37.7)$ \\
\hline$>19$ yrs & $319(60.4)$ \\
\hline \multicolumn{2}{|l|}{ Ethnicity } \\
\hline Han & $523(99)$ \\
\hline Tibetan & 2 \\
\hline Mongolian & 2 \\
\hline Huizu & 1 \\
\hline History of risk factors & $142(27)$ \\
\hline Familial MMD & 9 \\
\hline Hypertension & 45 \\
\hline Aneurysm & 16 \\
\hline Smoking or alcohol use & 47 \\
\hline Sinus arrhythmia & 20 \\
\hline Diabetes & 10 \\
\hline Type of hemorrhage & $196(37.1)$ \\
\hline $\mathrm{ICH}$ & 61 \\
\hline IVH & 7 \\
\hline $\mathrm{SAH}$ & 23 \\
\hline IVH w/ ICH & 33 \\
\hline Ischemic symptom & $332(62.9)$ \\
\hline TIA & 201 \\
\hline Acute stroke & 131 \\
\hline Surgical revascularization & 406 (76.9) \\
\hline STA-MCA anastomosis & 186 \\
\hline EDAS & 72 \\
\hline Multiple bur holes & 76 \\
\hline Carotid artery adventitial denudation & 46 \\
\hline Combined revascularization procedures & 26 \\
\hline
\end{tabular}

$\mathrm{ICH}=$ intracranial hemorrhage; $\mathrm{IVH}=$ intraventricular hemorrhage; $\mathrm{SAH}=$ subarachnoid hemorrhage.

new cases of MMD in our hospital, which may be a result of the increased number of MR or CT angiography examinations performed in patients who experienced relatively mild symptoms. Therefore, the exact incidence of MMD in China is not yet clear and might be even higher.

Some clinical features of MMD determined by this study differed from those in previous reports. First, epidemiological surveys in Japan, America, and Europe have shown that women have a higher incidence than men, with the female/male predominance ranging from 1.8:1 to $4.25: 1.2,3,9,16,17$ In the present study, the ratio of women to men was $0.87: 1$, which is much lower than the ratio reported for other Asian and Western countries. Several studies of Chinese patients with MMD also found that there is no difference in sex distribution. ${ }^{5,12,20}$ Second, the peaks for age distribution are somewhat different. MMD has been associated with age-specific infarct patterns. ${ }^{4}$ Two peaks for the age distribution of MMD have been demonstrated in Asia. In Japanese studies, the first peak age was be- tween 45 and 49 years, and the second was between 5 and 9 years; the peak for men was 10-14 years of age and for women was $20-24$ years. ${ }^{2,17}$ In this study, there were also 2 peaks for age distribution; however, the peak for adult patients was between 36 and 40 years, which is lower than that of the patients in Japan. For children, it was between 6 and 15 years, which is higher than that of the patients reported in Japanese series. We also found that the peaks for age distribution were similar between men and women. Third, the occurrence of familial MMD in our series $(1.7 \%)$ was much lower than that reported in other countries (approximately 5.6\%-15\%). However, we believe that because of the underdiagnosis of MMD in China currently, the incidence of familial MMD might have been underestimated.

Although hemorrhagic MMD was less frequent than the ischemic type, it was associated with higher morbidity and mortality rates. The reported mortality rates of initial intracranial hemorrhage in MMD have ranged from $6.8 \%$ to $17.9 \% .{ }^{19,26}$ The exact causes of bleeding in MMD remain unknown, but the rupture of microaneurysms in the collateral vessels at the base of the skull has been considered the main cause. ${ }^{10,13}$ Histopathological examination has shown that the walls of moyamoya collateral vessels are thinner and more brittle because of fibrin deposition, which is important for microaneurysm formation. ${ }^{1,8}$ In our cohort, only 16 patients (3\%) had aneurysms detected on angiography. We believe that many microaneurysms might not be visualized on angiograms. Most of the aneurysms occurred around the circle of Willis and peripheral collateral vessels, and the rate of aneurysm formation in patients with hemorrhagic MMD was higher than that in those with ischemic MMD. Clipping or endovascular treatment of aneurysm is thought to be meaningful in preventing hemorrhage in patients with MMD. However, fewer than half of our patients received surgery for aneurysm. The deep locations, small sizes, and unfavorable morphology of these microaneurysms are the reasons for the difficulty of surgical treatment. Furthermore, some of these aneurysms spontaneously resolved after bypass surgery, which has also been reported in other studies. ${ }^{15}$ Overall, for MMD associated with intracranial aneurysms, earlier surgical treatment can be performed if feasible, and, for the aneurysms not suitable for clipping or endovascular treatment, regular follow-up is recommended.

At present, surgical revascularization, which includes either direct STA-MCA bypass or indirect surgery, is the primary treatment for MMD, but both of these procedures are associated with complications. Symptomatic hyperperfusion, transient ischemic injury, postoperative hemorrhage, and seizures can occur after STA-MCA bypass. ${ }^{6,15}$ In this study, 13 (7\%) of 186 patients who received STAMCA procedures developed postoperative complications, which included 2 cerebral infarctions (1.1\%), 4 hemorrhages (2.2\%), 2 seizures (1.1\%), and 5 wound infections $(2.7 \%)$. Five subdural hygromas $(2.6 \%)$ and 4 seizures (2.1\%) occurred in 194 patients after indirect revascularization. Overall, the incidences of TIA, infarction, and hemorrhage were relatively low. We advocate careful measures, including monitoring of intraoperative and postoperative blood pressure fluctuations, blood flow changes, 
TABLE 2. Summary of baseline data for hemorrhagic and ischemic types of MMD

\begin{tabular}{|c|c|c|c|c|}
\hline \multirow[b]{2}{*}{ Baseline Data* } & \multicolumn{2}{|c|}{ Type of MMD } & \multirow[b]{2}{*}{$\mathrm{t} / \chi^{2}$ Test } & \multirow[b]{2}{*}{$\mathrm{p}$ Value } \\
\hline & Hemorrhagic $(n=196)$ & Ischemic ( $n=332)$ & & \\
\hline Mean age (yrs) & $31.42 \pm 10.80$ & $22.86 \pm 14.41$ & 7.19 & $<0.001$ \\
\hline Child/adult ratio & $28 / 168$ & $171 / 161$ & 71.65 & $<0.001$ \\
\hline Male/female ratio & $103 / 92$ & $179 / 154$ & 0.04 & 0.836 \\
\hline Mean DSA stage & $2.68 \pm 1.0$ & $2.58 \pm 0.97$ & 1.13 & 0.258 \\
\hline Family history (no.) & 5 & 4 & 1.36 & 0.243 \\
\hline Hypertension (no.) & 15 & 30 & 0.27 & 0.601 \\
\hline Diabetes (no.) & 4 & 6 & 0.04 & 0.839 \\
\hline Smoking or alcohol use (no.) & 23 & 24 & 3.19 & 0.07 \\
\hline Intracranial aneurysm (no.) & 13 & 3 & 13.91 & $<0.01$ \\
\hline Mean mRS score at admission & $2.18 \pm 1.21$ & $1.05 \pm 1.53$ & 2.45 & 0.02 \\
\hline Conservative treatment/revascularization ratio & $92 / 104$ & $30 / 302$ & 98.32 & $<0.01$ \\
\hline
\end{tabular}

and anesthesia delivery and application of intraoperative indocyanine green angiography and intraoperative electrophysiological examination.

Many studies have confirmed the role of surgical revascularization in the treatment of ischemic MMD. However, surgical management of hemorrhagic MMD is still controversial. The high associated rebleeding and mortality rates are the major causes of poor outcomes for patients with hemorrhagic MMD. 14,18,26 Therefore, reducing the occurrence of rebleeding is the most important consideration. In this study, 33 (9.9\%) of the followed-up 331 patients experienced intracranial hemorrhage, including 25 (41.7\%) of 60 conservatively treated patients and 8 (2.9\%) of 271 surgically treated patients. There was a significant difference in the rebleeding rates between patients in the surgical and the nonsurgical groups. However, a major limitation

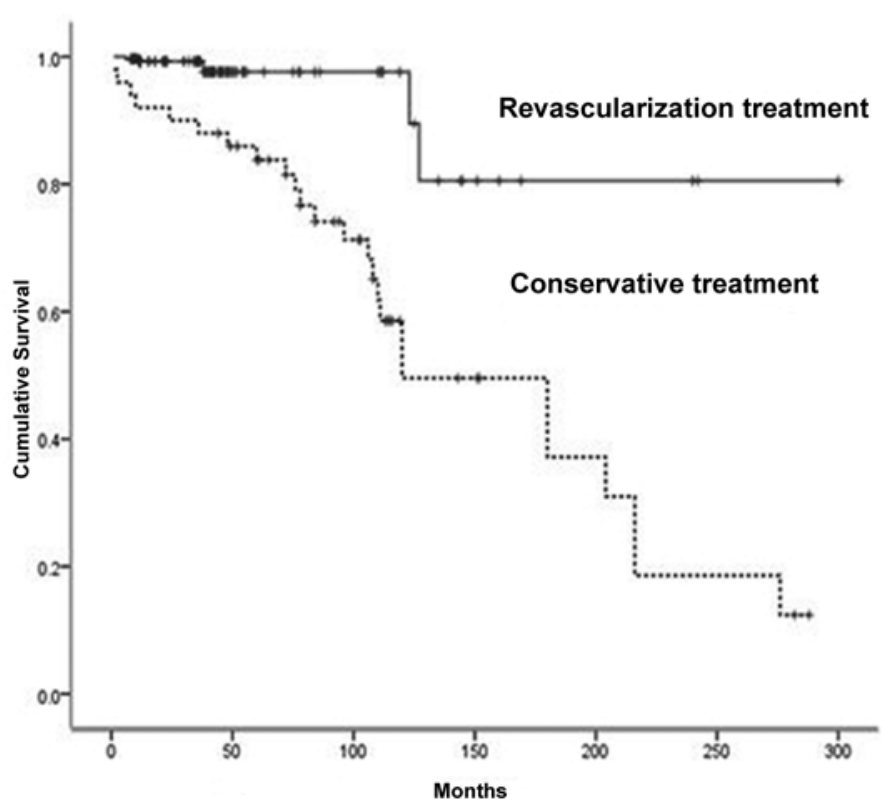

should be noted; all of the conservatively treated patients had hemorrhagic MMD, and patients with hemorrhagic MMD had a higher rebleeding rate than those with the ischemic type of MMD. In this series, 28 of 104 patients with hemorrhagic MMD experienced rebleeding, which was significantly higher than the rate in patients with ischemic MMD (26.9\% vs 2.2\%, respectively; Fig. 4 right).

Of the 104 patients, 25 of 60 (41.7\%) conservatively treated patients and 3 of $44(6.8 \%)$ surgically treated patients experienced a rebleeding event; the rebleeding rate in revascularization surgery was significantly lower than that in the conservative group. These results suggest that revascularization surgery may be of benefit for preventing rebleeding in patients with hemorrhagic MMD. A recent controlled trial also found that STA-MCA bypass provides a better preventive effect against rebleeding than does

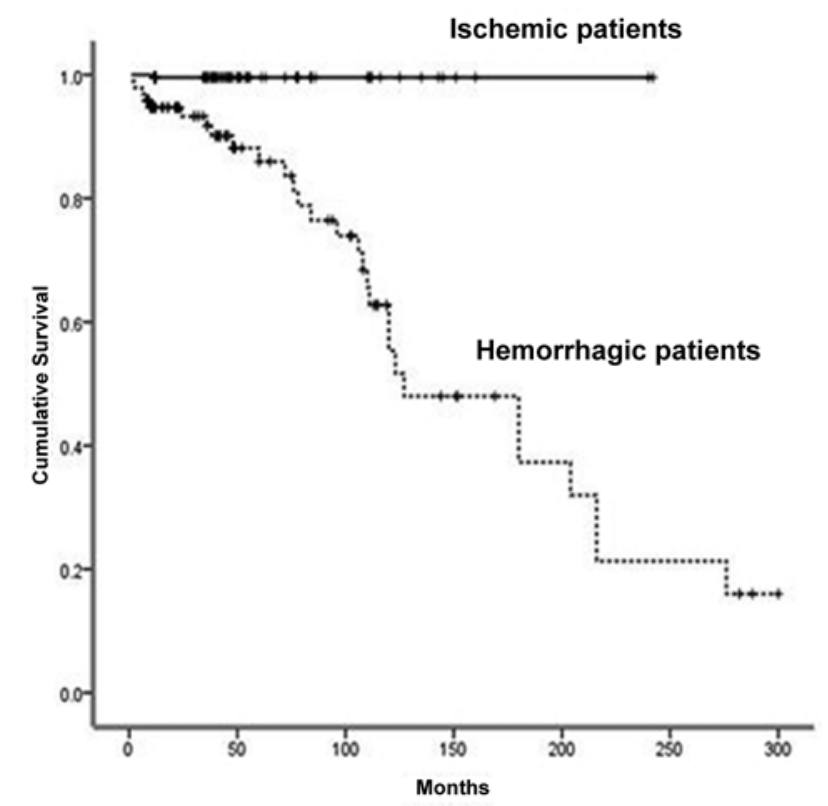

FIG. 4. Left: Kaplan-Meier cumulative hazard curve for rebleeding when comparing surgical and conservative treatment. Right: Kaplan-Meier cumulative hazard curve for rebleeding when comparing hemorrhagic and ischemic MMD. 
TABLE 3. Long-term outcomes of 331 patients with 1-year minimum follow-up

\begin{tabular}{|c|c|c|c|c|c|c|}
\hline \multirow[b]{2}{*}{ Outcome } & \multicolumn{2}{|l|}{ Treatment } & \multirow[b]{2}{*}{$p$ Value } & \multicolumn{2}{|c|}{ Initial Symptom } & \multirow[b]{2}{*}{$\mathrm{p}$ Value } \\
\hline & $\begin{array}{l}\text { Surgical (revascularization) } \\
\qquad(n=271)\end{array}$ & $\begin{array}{l}\text { Conservative } \\
(n=60)\end{array}$ & & $\begin{array}{l}\text { Hemorrhage } \\
(n=104)\end{array}$ & $\begin{array}{l}\text { Ischemia } \\
(n=227)\end{array}$ & \\
\hline \multicolumn{7}{|l|}{ Baseline data } \\
\hline Mean ( \pm SD) age (yrs) & $26 \pm 14$ & $29 \pm 10$ & 0.146 & $31 \pm 10$ & $24 \pm 14$ & $<0.001$ \\
\hline Male/female ratio & $133 / 138$ & $15 / 45$ & 0.001 & $33 / 71$ & $115 / 112$ & 0.001 \\
\hline Mean $\mathrm{mRS}$ score $( \pm \mathrm{SD})$ at admission & $1.89 \pm 1.03$ & $1.67 \pm 0.95$ & 0.134 & $1.58 \pm 1.07$ & $1.95 \pm 0.96$ & 0.020 \\
\hline Hypertension (no. [\%]) & $23(8.5)$ & $7(11.7)$ & 0.443 & $14(13.5)$ & $16(7.05)$ & 0.059 \\
\hline Intracranial aneurysm (no. [\%]) & $6(2.2)$ & $3(5)$ & 0.446 & $7(6.7)$ & $2(0.88)$ & 0.008 \\
\hline Smoking or alcohol use (no. [\%]) & $22(8.1)$ & $8(13.3)$ & 0.203 & $11(10.6)$ & $19(8.4)$ & 0.516 \\
\hline \multicolumn{7}{|l|}{ Follow-up events (no. [\%]) } \\
\hline New bleeding & $8(2.95)$ & $25(41.7)$ & $<0.001$ & $28(26.9)$ & $5(2.2)$ & $<0.001$ \\
\hline Death due to bleeding & $2(0.7)$ & $4(6.6)$ & 0.010 & $5(4.8)$ & $1(0.4)$ & 0.020 \\
\hline Ischemic event(s) & $51(18.8)$ & $17(28.3)$ & 0.099 & $21(20.2)$ & $47(20.7)$ & 0.915 \\
\hline \multicolumn{7}{|l|}{ Functional outcomes } \\
\hline Improvement in mRS score (no. [\%]) & $177(65.3)$ & $27(45)$ & 0.003 & $57(54.8)$ & $147(64.8)$ & 0.009 \\
\hline mRS Score 0-2 & $238(87.8)$ & $42(70.0)$ & 0.001 & $80(76.9)$ & $200(88.1)$ & 0.009 \\
\hline mRS Score 3-5 & $31(11.4)$ & $14(23.3)$ & 0.015 & $19(18.2)$ & $26(11.5)$ & 0.093 \\
\hline \multicolumn{7}{|l|}{ Perfusion (1 mo) } \\
\hline Increased (no./total no. [\%]) & $164 / 224(73.2)$ & $7 / 43(16.3)$ & $<0.001$ & $27 / 75(36)$ & $144 / 192(75)$ & $<0.001$ \\
\hline Decreased (no.) & 29 & 13 & 0.004 & 19 & 23 & 0.007 \\
\hline No change (no.) & 31 & 23 & $<0.001$ & 29 & 25 & $<0.001$ \\
\hline
\end{tabular}

conservative care for patients with hemorrhagic MMD. ${ }^{21}$ However, of our 196 patients with hemorrhagic MMD, nearly half did not receive revascularization surgery. Therefore, it is necessary to improve the rate of revascularization for patients with hemorrhagic MMD.

Several limitations of our outcome data must be noted. First, this study was a nonrandomized retrospective study, and the data were collected over 25 years, during which many diagnostic and therapeutic procedures had changed; therefore, selection bias may exist, and the results cannot completely reflect the epidemiology of MMD in China. Second, only $63 \%$ of the patients reached follow-up, and direct clinical follow-up was incomplete for all the patients. Third, it should be acknowledged that the comparison between the surgical group and the conservatively treated group is not scientific, and therefore the apparent advantages of surgery should be interpreted with caution. In addition, long-term follow-up angiographic data were not available for most of the patients who underwent surgical revascularization. Therefore, we were unable to make scientific cerebral blood flow comparisons between the various revascularization interventions. Furthermore, large randomized controlled trials comparing various revascularization interventions are warranted.

\section{Conclusions}

This study enhances our understanding of the clinical features, treatment, and long-term outcome of patients with MMD in mainland China. There was no difference in the sex distribution of Chinese patients with MMD, and 2 age peaks (6-15 and 36-40 years) were noted; ischemic
MMD was the main phenotypic presentation. Adult patients had a higher rate of hemorrhagic presentation than children. Hemorrhagic MMD had a much higher rate of rebleeding and poorer prognosis than the ischemic type. Surgical revascularization procedures can improve cerebral perfusion and have a positive impact on the prevention of rebleeding in patients with hemorrhagic MMD.

\section{References}

1. Achrol AS, Guzman R, Lee M, Steinberg GK: Pathophysiology and genetic factors in moyamoya disease. Neurosurg Focus 26(4):E4, 2009

2. Baba T, Houkin K, Kuroda S: Novel epidemiological features of moyamoya disease. J Neurol Neurosurg Psychiatry 79:900-904, 2008

3. Chiu D, Shedden P, Bratina P, Grotta JC: Clinical features of moyamoya disease in the United States. Stroke 29:13471351,1998

4. Cho HJ, Jung YH, Kim YD, Nam HS, Kim DS, Heo JH: The different infarct patterns between adulthood-onset and childhood-onset moyamoya disease. J Neurol Neurosurg Psychiatry 82:38-40, 2011

5. Duan L, Bao XY, Yang WZ, Shi WC, Li DS, Zhang ZS, et al: Moyamoya disease in China: its clinical features and outcomes. Stroke 43:56-60, 2012

6. Fujimura M, Mugikura S, Kaneta T, Shimizu H, Tominaga T: Incidence and risk factors for symptomatic cerebral hyperperfusion after superficial temporal artery-middle cerebral artery anastomosis in patients with moyamoya disease. Surg Neurol 71:442-447, 2009

7. Fukui M: Guidelines for the diagnosis and treatment of spontaneous occlusion of the circle of Willis ('moyamoya' disease). Research Committee on Spontaneous Occlusion of the Circle of Willis (Moyamoya Disease) of the Ministry 
of Health and Welfare, Japan. Clin Neurol Neurosurg 99 (Suppl 2):S238-S240, 1997

8. Fukui M, Kono S, Sueishi K, Ikezaki K: Moyamoya disease. Neuropathology 20 (Suppl):S61-S64, 2000

9. Hallemeier CL, Rich KM, Grubb RL Jr, Chicoine MR, Moran CJ, Cross DT III, et al: Clinical features and outcome in North American adults with moyamoya phenomenon. Stroke 37:1490-1496, 2006

10. Hamada J, Hashimoto N, Tsukahara T: Moyamoya disease with repeated intraventricular hemorrhage due to aneurysm rupture. Report of two cases. J Neurosurg 80:328-331, 1994

11. Han DH, Kwon OK, Byun BJ, Choi BY, Choi CW, Choi JU, et al: A co-operative study: clinical characteristics of 334 Korean patients with moyamoya disease treated at neurosurgical institutes (1976-1994). Acta Neurochir (Wien) 142:1263-1274, 2000

12. Hung CC, Tu YK, Su CF, Lin LS, Shih CJ: Epidemiological study of moyamoya disease in Taiwan. Clin Neurol Neurosurg 99 (Suppl 2):S23-S25, 1997

13. Iwama T, Morimoto M, Hashimoto N, Goto Y, Todaka T, Sawada M: Mechanism of intracranial rebleeding in moyamoya disease. Clin Neurol Neurosurg 99 Suppl 2:S187S190, 1997

14. Kawaguchi S, Okuno S, Sakaki T: Effect of direct arterial bypass on the prevention of future stroke in patients with the hemorrhagic variety of moyamoya disease. J Neurosurg 93:397-401, 2000

15. Kim DS, Kang SG, Yoo DS, Huh PW, Cho KS, Park CK: Surgical results in pediatric moyamoya disease: angiographic revascularization and the clinical results. Clin Neurol Neurosurg 109:125-131, 2007

16. Kraemer M, Heienbrok W, Berlit P: Moyamoya disease in Europeans. Stroke 39:3193-3200, 2008

17. Kuriyama S, Kusaka Y, Fujimura M, Wakai K, Tamakoshi A, Hashimoto S, et al: Prevalence and clinicoepidemiological features of moyamoya disease in Japan: findings from a nationwide epidemiological survey. Stroke 39:42-47, 2008

18. Liu X, Zhang D, Shuo W, Zhao Y, Wang R, Zhao J: Long term outcome after conservative and surgical treatment of haemorrhagic moyamoya disease. J Neurol Neurosurg Psychiatry 84:258-265, 2013

19. Matsushima T, Inoue T, Suzuki SO, Fujii K, Fukui M, Hasuo $\mathrm{K}$ : Surgical treatment of moyamoya disease in pediatric patients-comparison between the results of indirect and direct revascularization procedures. Neurosurgery 31:401-405, 1992
20. Miao W, Zhao PL, Zhang YS, Liu HY, Chang Y, Ma J, et al: Epidemiological and clinical features of Moyamoya disease in Nanjing, China. Clin Neurol Neurosurg 112:199-203, 2010

21. Miyamoto S, Yoshimoto T, Hashimoto N, Okada Y, Tsuji I, Tominaga T, et al: Effects of extracranial-intracranial bypass for patients with hemorrhagic moyamoya disease: results of the Japan Adult Moyamoya Trial. Stroke 45:1415-1421, 2014

22. Suzuki J, Takaku A: Cerebrovascular "moyamoya" disease. Disease showing abnormal net-like vessels in base of brain. Arch Neurol 20:288-299, 1969

23. Suzuki J, Takaku A, Kodama N, Sato S: An attempt to treat cerebrovascular 'Moyamoya' disease in children. Childs Brain 1:193-206, 1975

24. Takeuchi K, Shimizu K: Hypoplasia of the bilateral internal carotid arteries. Brain Nerve 9:37-43, 1957

25. Wakai K, Tamakoshi A, Ikezaki K, Fukui M, Kawamura T, Aoki R, et al: Epidemiological features of moyamoya disease in Japan: findings from a nationwide survey. Clin Neurol Neurosurg 99 (Suppl 2):S1-S5, 1997

26. Yoshida Y, Yoshimoto T, Shirane R, Sakurai Y: Clinical course, surgical management, and long-term outcome of moyamoya patients with rebleeding after an episode of intracerebral hemorrhage: an extensive follow-up study. Stroke 30:2272-2276, 1999

\section{Author Contributions}

Conception and design: JZ Zhao, Liu, Zhang, S Wang. Acquisition of data: Liu. Analysis and interpretation of data: Liu, S Wang, Y Zhao, Teo, R Wang, Cao, Ye, Kang. Drafting the article: Liu. Critically revising the article: JZ Zhao, Zhang, Y Zhao, Teo, R Wang. Reviewed submitted version of manuscript: JZ Zhao, Zhang. Approved the final version of the manuscript on behalf of all authors: JZ Zhao. Statistical analysis: Liu. Administrative/technical/material support: Zhang. Study supervision: JZ Zhao, Zhang.

\section{Correspondence}

Ji-Zong Zhao, Department of Neurosurgery, Beijing Tiantan Hospital, Capital Medical University, 6 Tiantanxili, Chongwen District, Beijing 100050, China. email: zhaojz205@gmail.com. 